\title{
NOTICIAS \\ 84 \\ XXVIII \\ Jornadas \\ Odontológicas Estudiantiles de la Facultad de Odontología
}

$S_{2}$ llevaron a cabo el 19 y 20 de septiembre de 20I3, las XXVIII Jornadas Odontológicas Estudiantiles de la Facultad de Odontología de la Universidad Nacional del Nordeste.

El acto de apertura se desarrollo en el Salón Auditorium y durante el mismo hicieron uso de la palabra, el Presidente del Centro de Estudiantes de la Facultad, Srta. Flavia Bedoya, el Secretario de Asuntos Estudiantiles, Od. Juan Manuel Gómez y para finalizar dejando inaugurada las Jornadas, el Sr. Decano de la Facultad Prof. Roque Oscar Rosende.

Posteriormente $y$ en el marco del acto inaugural, se vivió la emocionante entrega de Medallas de Oro al mejor promedio de la Promoción 2012, Od. Pablo Javier Angeletti. También tuvo su reconocimiento la Srta. Marina Laura Kees quien recibió la Medalla de Plata "Premio Facultad de Odontología UNNE, al esfuerzo y voluntad - periodo lectivo 2012".

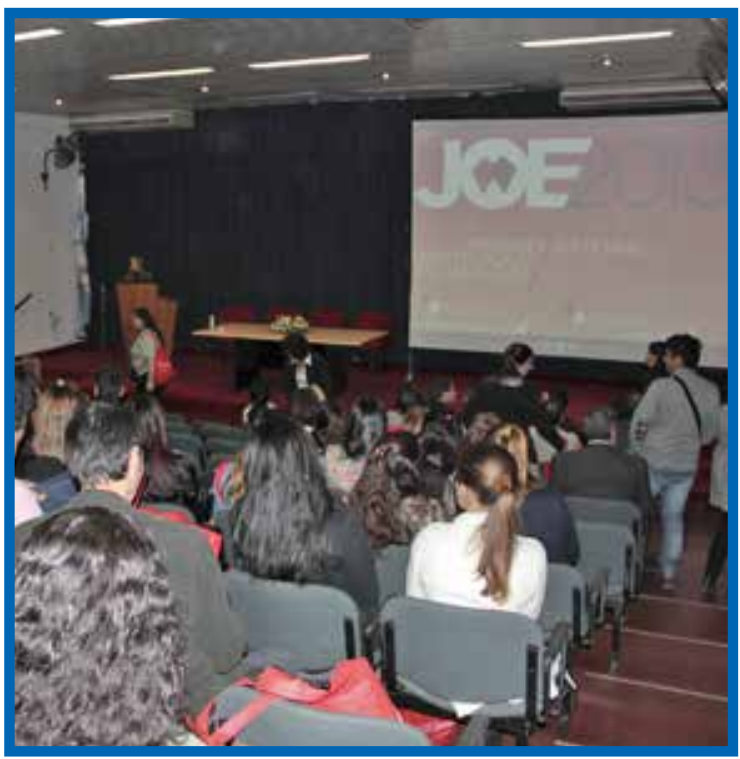

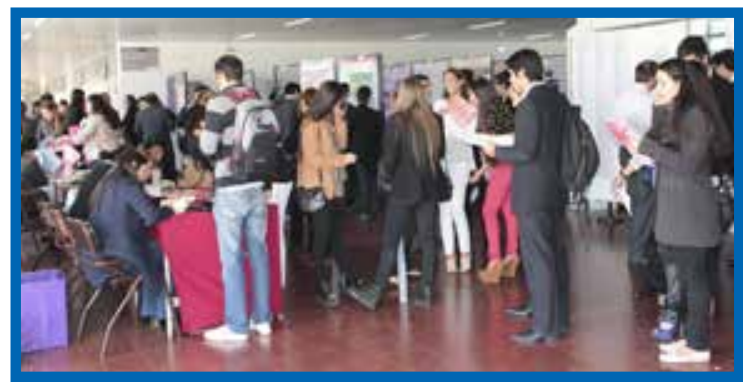

Las Jornadas estudiantiles representan un espacio científico de intercambio y socialización que propicia la presentación en conferencias o en sistema póster de trabajos disciplinares de las distintas asignaturas realizados por los estudiantes, con el asesoramientos de los docentes y la coordinación de los Profesores Titulares y Adjuntos.

En el marco de las mismas se desarrolló el Curso "Tratamiento de las Patologías Buco Máxilo Faciales y su Profilaxis Antibiótica" a cargo del Dr. Guillermo Rey.

En el Hall de la Facultad se exhibieron los posters. Esta modalidad permitió la participación de alumnos, presentando temas de actualidad, casos clínicos, experiencias educativas y de extensión realizadas durante el presente año lectivo.

Asimismo, en los Salones Auditorios A y B, se realizaron las disertaciones a cargo de los grupos de exposición, conformados por no más de tres alumnos con el asesoramiento de dos docentes. Desde las últimas Jornadas Odontológicas Estudiantiles, se viene realizando un reconocimiento especial a estudiantes y docentes con la premiación de los cinco mejores trabajos de las categorías póster y conferencia. El jurado conformado por docentes de reconocida trayectoria de la unidad académica, tuvieron la difícil tarea de seleccionar a los diez mejores trabajos. 


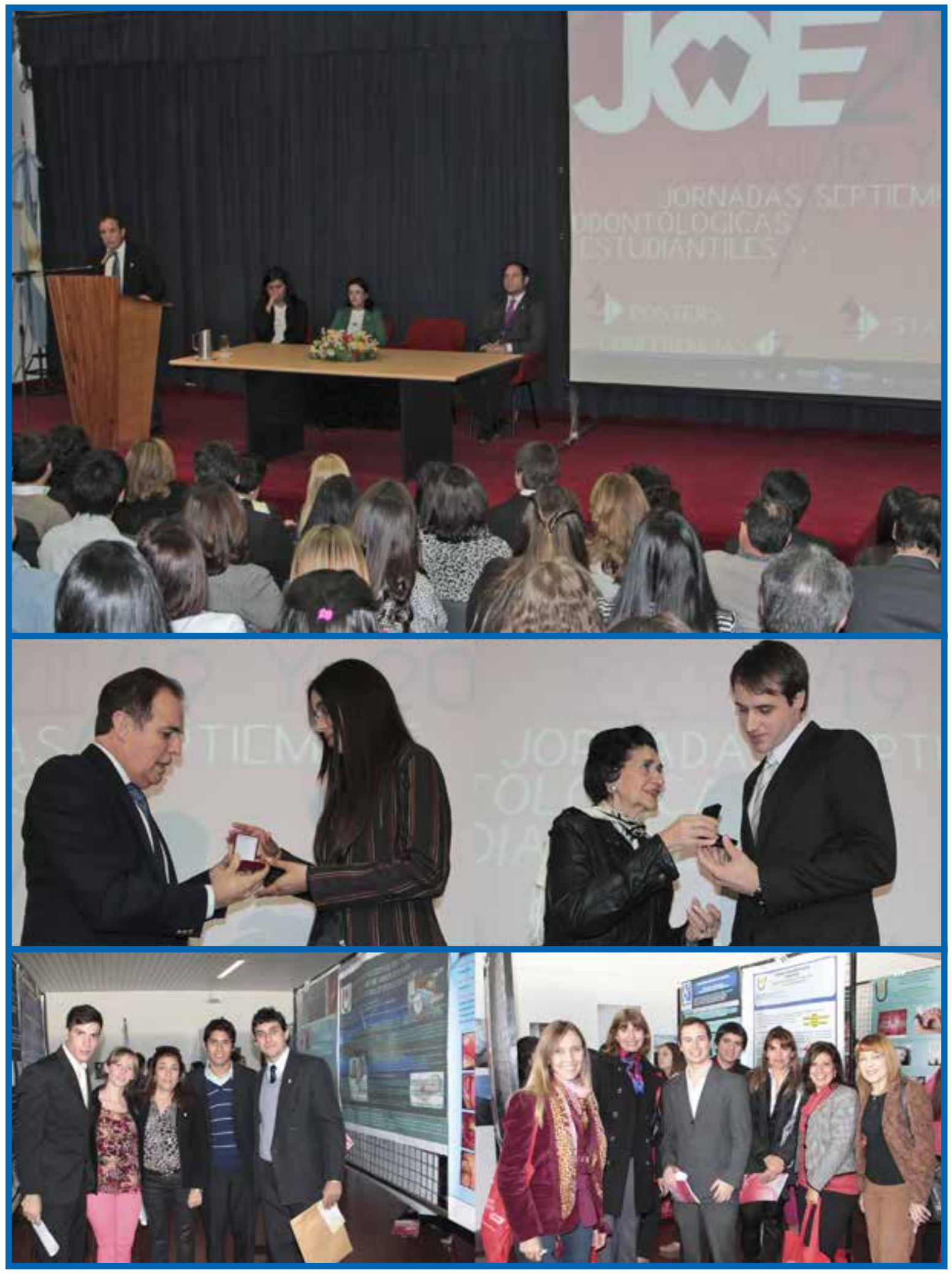




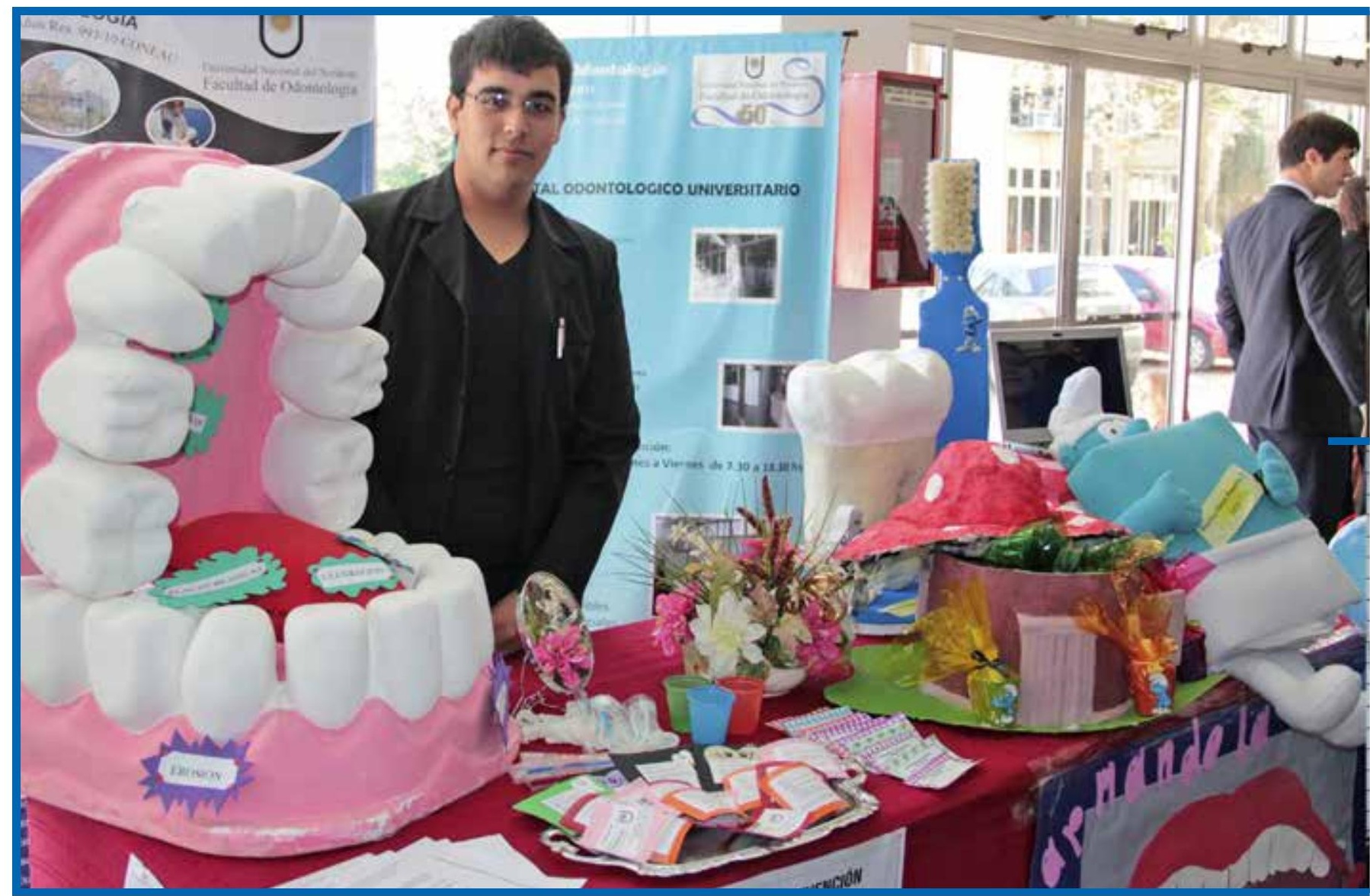

\section{MODALIDAD PÓSTER}

\section{"AUTOEXAMEN BUCAL"}

Autores: María Lourdes Strumia. Araceli Torres. Gabriela Varela. María Victoria Valdez. Docentes Asesores: Od. Elena Soledad Ortiz Barreto. Od. Claudio Alejandro Modenutti. Docente Coordinador: Prof. María Cristina Samela. Prof. Víctor Ricardo Fernández.

\section{“TRATAMIENTO ODONTOLÓGICO EN NIÑOS CON SÍNDROMES DEL ESPEC-} TRO AUTISTA"

Autores: Rocío Anahí Piris. Luis Américo Maciel. Docente Asesor: Roxana Lilian Blanco. Docente Coordinador: Prof. María Cristina Ojeda

\section{"CÉlULAS Y TEJIDOS, JUNTOS A LA PAR"}

Autores: Florencia Valsangiacomo. Constanza Aranguiz. María Florencia Brenn. María Lourdes Aquino. Docente Asesor: Od. Melisa Lezcano. Docente Coordinador: Prof. María Alejandra Gili

\section{"LA NANOTECNOLOGÍA Y LAS RESINAS REFORZADAS "}

Autores: Emilia lurinic, Maria Emilia Omastoff. Docentes Asesores: Od. Ana María Slobayen, Od. María Rosana Ascona. Docente Coordinador: Prof. María Eugenia Zamudio.

INFORME PERICIAL: UN CASO CLINICO"

Autores: María Victoria Formichelli, Emilia Marisel Gallardo. Docente Asesor: Prof. Olga Leonor Ariasgago. María del Carmen Scetti. Docente Coordinador: Prof. José R. Rivarola. 


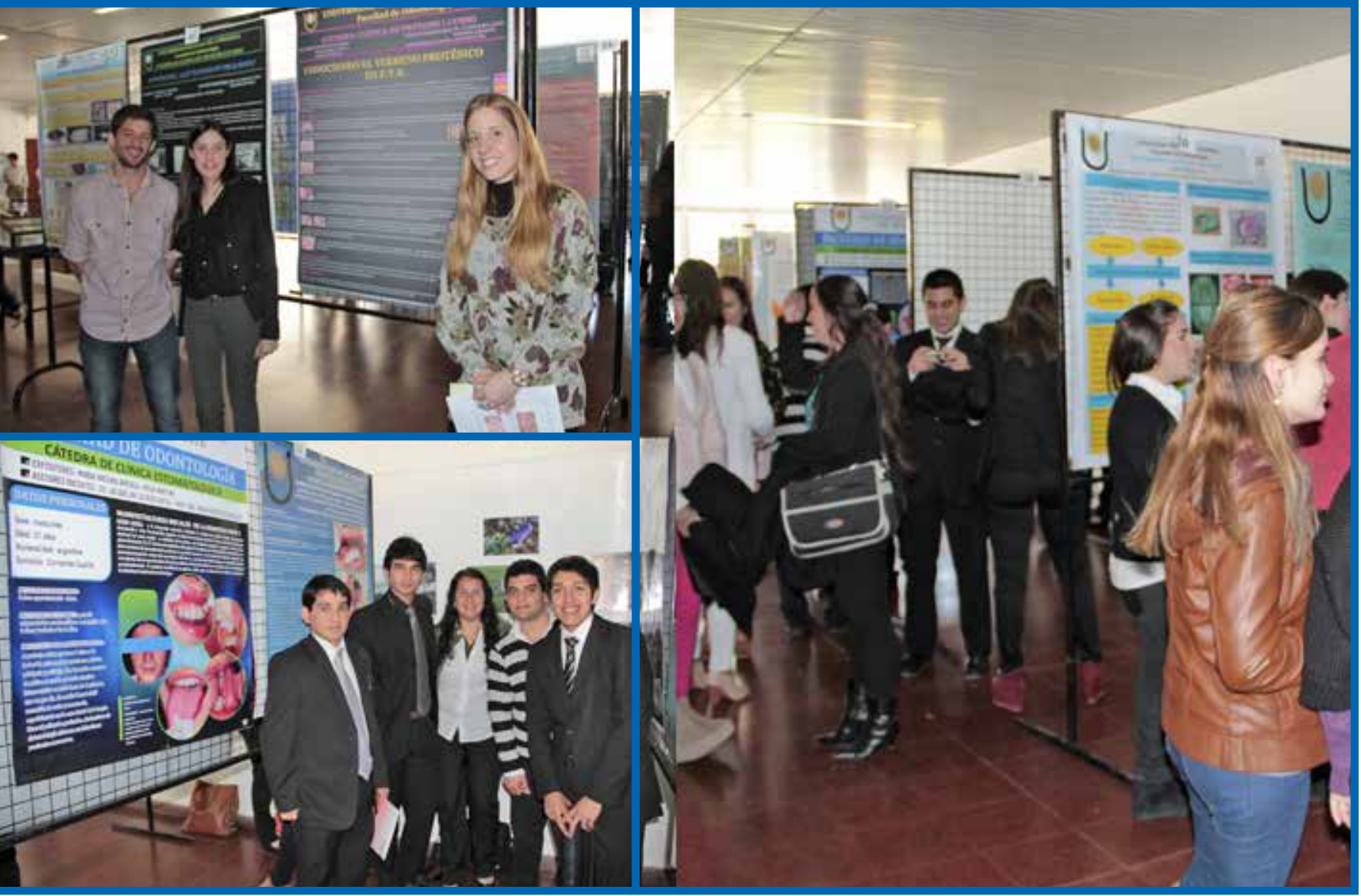

SISTEMA DE CONFERENCIAS

\section{"TÉCNICA DE GLASEADO EN CERÁMICA DENTAL"}

Autores: Rudy Xiscati. Rony Xiscati. Seyla Belledonne Sosa. Cátedra: Biomateriales. Docentes Asesores: Od. Dario Ammatuna. Od. Claudia A. Modenutti. Docente Coordinador: Prof. Maria Eugenia Zamudio.

"IMPORTANCIA FISIOLÓGICA Y ANATÓMICA DEL NERVIO HIPOGLOSO Y SUS DIVERSAS AFECCIONES."

Autores: Matías Augusto Dreher. Ricardo Ariel Escalante. Cátedras: Fisiología Humana. Anatomía General. Docentes Asesores: Od. Jorge Orlando Ponce. Od. Juan Manuel Gómez. Docentes Coordinadores: Prof. Rolando Pablo Juarez. Prof. María H. Frank.

“INJERTOS OSEOS AUTOLOGOS"

Autores: Carlos Javier Aquino. Marina Benítez Alarcón. Cátedra: Cirugía III: Cirugía y Traumatología Bucomaxilofacial. Docentes Asesores: Od. Sebastián Krupp. Luis Guillermo Carrio Hardy. Docente Coordinador: Prof. Roque Oscar Rosende.

\section{"CARACTERÍSTICAS DEL SBS"}

Autor: Pablo Vierci, Celeste Streuli. Docente Asesor. Od. Norma Beatriz Silva. Docente Coordinador: Prof. José Roberto Rivarola.

"REABSORCIÓN RADICULAR: DIAGNOSTICO Y TRATAMIENTO"

Autores: María Alejandra Fernández Pedro. Emilia Marisel Gallardo. Docente Coordinador: Od. Ricardo Pacella. 